\title{
COMPACTNESS CRITERIA FOR SPACES OF MEASURABLE FUNCTIONS
}

\author{
YU. BRUDNYI
}

\begin{abstract}
The paper contains new compactness criteria for a wide class of translation-invariant spaces of measurable functions. The results imply new compactness theorems for the families of Orlicz classes (such as $L_{0}\left(\mathbb{R}^{d}\right)$ ) and MarcinkiewiczLorentz spaces (including $L_{p q}$ with $p<1$ ).
\end{abstract}

\section{INTRODUCTION}

In this paper, we intend to establish Arcela-Ascoli type compactness criteria for a wide class of non-Banach function lattices on $\mathbb{R}^{d}$. Compactness has been used in so many problems of analysis that its importance is out of question. However, the choice of a field looking exotic for most analysts should somehow be motivated. The simplest illustration of encountering non-Banach spaces in classical analysis is a result concerning the long-standing Kolmogorov rational approximation problem [2]. To formulate the problem, we denote by $r_{n}(f)$ the best approximation of $f$ in $C[0,1]$ by fractions $p / q$ where $p, q$ are polynomials of degree $n$.

Problem. Characterize the function class $\mathcal{R}_{\lambda}, \lambda>0$, given by the finiteness of

$$
|f|_{\mathcal{R}_{\lambda}}:=\sup _{n \geq 1} n^{\lambda} r_{n}(f) .
$$

A partial solution asserts that for every $\varepsilon>0$ and $\frac{1}{p}=\lambda$ we have

$$
c_{1}(\varepsilon)|f|_{\operatorname{Lip}(\lambda, p-\varepsilon)} \leq|f|_{\mathcal{R}_{\lambda}} \leq c_{2}(\varepsilon)|f|_{\operatorname{Lip}(\lambda, p+\varepsilon)}
$$

with constants tending to infinity as $\varepsilon \rightarrow 0$.

Here $\operatorname{Lip}(\lambda, q), \lambda>0,0<q \leq \infty$, is defined in terms of the $k$-modulus of continuity with $k>\lambda$, i.e.,

$$
|f|_{\operatorname{Lip}(\lambda, q)}:=\sup _{t>0} \frac{\omega_{k}(f ; t)_{L_{q}(0,1)}}{t^{\lambda}} .
$$

Note that for $\lambda>1$ the exponent $p:=\lambda^{-1}$ is less than 1, i.e., quasi-Banach spaces arise naturally in the answer. The starting point of the proof in [2] is the embedding

$$
\operatorname{Lip}(\lambda, q) \subset C[0,1], \quad \lambda>\frac{1}{q},
$$

which is well known for $q \geq 1$ but requires a proof for $q<1$.

For the time being, the only known proof is based on an approximation inequality from [3], named there after Whitney. (The name seems to be commonly adopted; see, e.g., [17], where Subsection 6.4 titled "Two Whitney's theorems" contains instead two present authors' results.)

2010 Mathematics Subject Classification. Primary 46E30.

Key words and phrases. Compactness, translation invariant $F$ - and B-lattices, Orlicz classes, Marcinkiewicz-Lorentz quasi-Banach spaces. 
The proof of Whitney's type inequalities for Banach spaces is based on construction of linear operators onto the spaces of polynomials of fixed degree. However, in the non-Banach case there are no linear operators of finite rank. The only known proof is nonconstructive and exploits an Arcela-Ascoli type compactness theorem for quasiBanach spaces (it was presented in [4]; see also the Appendix).

Finally, replacing $\left\{n^{\lambda}\right\}$ in Kolmogorov's problem by more general majorants and using the approach discussed, we encounter similar compactness problems for other quasiBanach spaces (Orlicz, Lorentz, etc.).

This leads to the following problem studied in this paper 1

Let $X$ be a translation-invariant quasi-Banach lattice on $\mathbb{R}^{d}$.

Problem. Prove that a subset of $X$ is precompact if it is bounded and equicontinuous.

The problem is still open but we present a positive answer for a wide class of lattices including weighted Marcinkiewicz-Lorentz spaces and Orlicz spaces (even for FréchetOrlicz spaces). The proof is based on compactness results concerning two opposite general classes of lattices, Fréchet and Banach.

The paper consists of four sections, the first of which presents the basic notions and results of the Fréchet lattice (briefly, F-lattice) theory required in the paper. Though the standard textbooks on the subject concern only the Banach lattice theory, the proofs there can trivially be adapted to the required $F$-lattice case. The next two sections contain, respectively, the main results and their proofs. The final section discusses the known Arcela-Ascoli type results for spaces of measurable functions and their relationship with the results of the present paper.

\section{§1. Preliminaries}

An F-space is defined by the very same axioms as those of a Banach space, with the homogeneity norm axiom replaced by

$$
\|-x\|=\|x\|
$$

See, e.g., [5, Chapter 2] for the details.

An $F$-lattice $(X,\|\cdot\|)$ on a measure space $(\Omega, \mu)$ with a $\sigma$-finite measure $\mu$ is an $F$-space whose $F$-norm satisfies

$$
|f| \leq|g|, \quad g \in X \Rightarrow f \in X \quad \text { and } \quad\|f\| \leq\|g\| .
$$

All inequalities and identities in $X$ are understood $\mu$-almost everywhere (briefly, $\bmod \mu$ or, for the Lebesque measure on $\mathbb{R}^{d}$, a.e.).

A subset $S \subset \Omega$ is the support of $X$ (denoted by $\operatorname{supp} X)$ if $S$ is a $\bmod \mu \operatorname{minimal}$ $\mu$-measurable set containing all sets $\{\omega \in \Omega: f(\omega) \neq 0\}, f \in X$; see [11, Subsection IV.3.2] for its existence and uniqueness.

Example 1.1. A primary example of an $F$-lattice is the linear space of $\mu$-measurable functions on $\Omega$ whose $F$-norm is given by

$$
\|f\|_{0}:=\int_{\Omega} \frac{|f|}{1+|f|} w d \mu
$$

here $w$ is a $\mu$-measurable function such that

$$
w>0 \text { and } \int_{\Omega} w d \mu<\infty .
$$

We take $w=1$ if $\mu(\Omega)<\infty$.

\footnotetext{
${ }^{1}$ See $\S 1$ for the definitions of the notions appearing below.
} 
Convergence in this $F$-norm is equivalent to convergence in $\mu$-measure, i.e., $f_{n} \rightarrow f$ if for every $S \subset \Omega$ with $\mu(S)<\infty$ and every $\varepsilon>0$ we have

$$
\lim _{n \rightarrow \infty}\left\{\omega \in \Omega:\left|f-f_{n}\right|(\omega)>\varepsilon\right\}=0 .
$$

The next result known for the Banach case is also true in our setting, because the proof for the former case is easily adapted to F-lattices; see, e.g., [11, Subsection II.3].

Proposition 1.2. Let $X$ be an F-lattice on $(\Omega, \mu)$. The following is true.

(a) $X$ is continuously embedded into $L_{0}(\Omega, \mu)$.

(b) There exists an increasing sequence $\left\{S_{n}\right\}$ of sets of finite $\mu$-measure such that

$$
\bigcup S_{n}=\operatorname{supp} X \text { and } \mathbb{1}_{S_{n}} \in X \text { for all } n \text {. }
$$

Hereafter, $\mathbb{1}_{S}$ stands for the indicator of $S$.

In examples below, we encounter quasi-F-lattices whose definition differs only at one point: instead of the triangle inequality, an $F$-quasinorm satisfies

$$
\|f+g\| \leq K(\|f\|+\|g\|)
$$

for a fixed $K>1$ and all $f, g$.

All compactness results of the present paper are valid for these objects due to the following statement.

Proposition 1.3. Let $(X,\|\cdot\|)$ be a quasi-F-lattice satisfying (1.3). There exists a lattice $F$-norm $\|\cdot\|^{*}$ on $X$ such that for every $f \in X$ and some $0<p<1$ we have

$$
\|f\|^{*} \leq\|f\|^{p} \leq 4\|f\|^{*} .
$$

Proof. Following the Frink construction [7] introduced originally for quasimetric spaces, we set

$$
\|f\|^{*}:=\inf \sum\left\|f_{j}\right\|^{p}
$$

for $f \in X$, where $p:=\left(1+\log _{2} K\right)^{-1}$ and the infimum is taken over all finite decompositions $f=\sum f_{j}$.

By Frink's theorem, (1.4) holds true for $\|f\|^{*}$. It is easily seen that $\|\cdot\|^{*}$ is a complete $F$-norm.

It remains to prove the monotonicity of $\|\cdot\|^{*}$. By the definition, it suffices to prove (1.1) for $0 \leq g \leq f \in X$. However, for $f \geq 0$ the infimum in (1.5) can only be taken over the decompositions with $f_{j} \geq 0$. The decomposition property of lattices, see, e.g., [16. II.1a] implies that for every $f=\sum f_{j}$ with $f_{j} \geq 0$ there exist $g_{j}$ 's with $0 \leq g_{j} \leq f_{j}$ and $g=\sum g_{j}$. Therefore,

$$
\|g\|^{*} \leq \sum\left\|g_{j}\right\|^{p} \leq \sum\left\|f_{j}\right\|^{p}
$$

and (1.1) follows.

Remark 1.4. It is readily seen that, in the special case of quasi-Banach lattices, the $F$-norm defined by (1.5) satisfies

$$
\|\lambda f\|^{*}=|\lambda|^{p}\|f\|^{*}
$$

for every $\lambda \in \mathbb{R}$ and $f \in X$. Hence, in this case, $\left(X,\|\cdot\|^{*}\right)$ is a so-called $p$-Banach lattice.

Definition 1.5. A (quasi-) $F$-lattice $(X,\|\cdot\|)$ on $(\Omega, \mu)$ has absolutely continuous $F$-norm if for every $f \in X$ and every sequence $\left\{S_{n}\right\}$ of $\mu$-measurable subsets of $\Omega$ with empty intersection we have

$$
\lim _{n \rightarrow \infty}\left\|f \cdot \mathbb{1}_{S_{n}}\right\|=0 .
$$


Example 1.6. (a) Let $N$ be a homeomorphism of $\mathbb{R}_{+}$equal to 0 at 0 and satisfying the $\Delta_{2}$-condition, i.e.,

$$
\Delta_{N}:=\sup _{t>0} \frac{N(2 t)}{N(t)}<\infty .
$$

By definition, the Orlicz class $N(L)$ is the set of $\mu$-measurable functions on $\Omega$ satisfying

$$
\rho_{N}(f):=\int_{\Omega} N(|f|) d \mu<\infty .
$$

By (1.7), $N(L)$ is a linear space. This and the monotonicity of $N$ also imply that

$$
\rho_{N}(f+g) \leq\left(\Delta_{N}\right)^{2}\left(\rho_{N}(f)+\rho_{N}(g)\right),
$$

i.e., $\rho_{N}$ is a monotone $F$-quasinorm. Moreover, $\left(N(L), \rho_{N}\right)$ is complete, see, e.g., [21, Subsections 1.3 and 1.5].

(b) Under certain additional conditions on $N$, the space $N(L)$ is quasi-Banach. To state these conditions, for $t>0$ we set

$$
n(t):=\inf \left\{\lambda>0 ; N(\lambda t) \geq \frac{N(t)}{2}\right\} .
$$

Then the Rolewicz theorem [21, III.3] asserts that $N(L)$ is a quasi-Banach space if

$$
\inf _{t>0} n(t)>0 .
$$

(c) If $N$ is convex, $N(L)$ can be equipped with the following Banach norm equivalent to $\rho_{N}$ :

$$
\|f\|:=\inf \left\{\lambda>0 ; \rho_{N}\left(\frac{|f|}{\lambda}\right) \leq 1\right\} .
$$

This space is denoted by $L_{N}$ and named an Orlicz space; see, e.g., 14.

(d) Finally, we consider one more family of quasi-Banach lattices on $(\Omega, \mu)$, the Marcinkiewicz-Lorentz spaces $L_{p, N}$, where $0<p \leq \infty$ and $N: \mathbb{R}_{+} \rightarrow \mathbb{R}_{+}$is the same as in (a).

The corresponding quasinorm is given by

$$
\|f\|_{p, N}:=\left\{\int_{\mathbb{R}_{+}}\left(f^{*}(t) N(t)\right)^{p} \frac{d t}{t}\right\}^{\frac{1}{p}}
$$

here $f^{*}$ is the nonincreasing rearrangement of $f$.

For $N(t):=t^{\frac{1}{q}}, 0<q \leq \infty$, this is named a Lorentz space and denoted by $L_{p q}$.

At least for $L_{p q}$ with $1<p<\infty$ and $1 \leq q<\infty$ (the Banach case), it is known that $L_{p q}$ is complete; see, e.g., [15, II.5]. It can easily be checked that a slight modification of the proof given there establishes the same facts in the general case.

Finally, we note that the families in (a) and (c) contain the scale $\left\{L_{p}\right\}_{0<p \leq \infty}$.

Since the basic compactness concept, equicontinuity, measures the behavior of the modulus of continuity for sets of functions in $X$, its introduction requires some additional structure. The simplest is to choose $\mathbb{R}^{d}$ with the Lebesgue $d$-measure as a measure space.

Hereafter $S$ denotes a (Lebesgue) measurable subset of $\mathbb{R}^{d}$ and $|S|$ stands for its measure.

In what follows, we restrict our consideration to the class of translation-invariant lattices on $\mathbb{R}^{d}$. Here is the definition.

Definition 1.7. An F-lattice $X$ on $\mathbb{R}^{d}$ is said to be translation-invariant if the shift operators

are isometries of $X$.

$$
\tau_{y}: f \mapsto f(\cdot+y), \quad y \in \mathbb{R}^{d},
$$


Clearly, $N(L)$ belongs to this class and the same is true for $L_{p, N}$, because by the definition of the rearrangement we have $\left(\tau_{y} f\right)^{*}=f^{*}, y \in \mathbb{R}^{d}$.

In the remaining part of the paper, we deal with $F$-lattices on $\mathbb{R}^{d}$ subject to Definitions 1.5 and 1.7. For convenience, we combine them as follows.

Definition 1.8. The class of $F$-lattices on $\mathbb{R}^{d}$ whose $F$-norm is translation-invariant and absolutely continuous is denoted by $\mathcal{L}$ (after Lebesgue).

By the Lebesgue convergence theorem, $N(L)$ and $L_{p, N}$ with $p<\infty$ belong to $\mathcal{L}$.

Definition 1.9. A function $f$ is continuous in a translation-invariant $F$-lattice $(X,\|\cdot\|)$ on $\mathbb{R}^{d}$ if

$$
\lim _{y \rightarrow 0}\left\|\tau_{y} f-f\right\|=0 .
$$

The set of all functions continuous in $X$ is denoted by $X^{0}$. It is a closed linear subspace in $X$ but, in general, is not an $F$-lattice.

Proposition 1.10. If $X \in \mathcal{L}$, then $X=X^{0}$.

Proof. It suffices to find a subset of $X^{0}$ dense in $X$. By the separability of the Lebesgue measure, finite linear combinations of indicators $\mathbb{1}_{S}$ with $|S|<\infty$ and $\mathbb{1}_{S} \in X$ are dense in $X$; see, e.g., Lemma 3 in [11, IV.3.2]. To show that this set is contained in $X^{0}$, it suffices to check that $f:=\lambda \cdot \mathbb{1}_{S}$ is contained in $X^{0}$ for $\lambda \in \mathbb{R},|S|<\infty$. But for this $f$ we have

$$
\left\|\tau_{y} f-f\right\|=\left\|\lambda \cdot \mathbb{1}_{S \triangle\{S+y\}}\right\|
$$

and the measure of the symmetric difference $|S \triangle\{S+y\}|$ tends to 0 as $y \rightarrow 0$. Since $\|\cdot\|$ is absolutely continuous, the right-hand side of (1.13) tends to 0 as $y \rightarrow 0$.

Remark 1.11. Denoting by $X^{+}$the $F$-sublattice of $X$ consisting of functions with absolutely continuous norm, we conclude that $X^{+} \subset X^{0}$. However, the former space may be essentially smaller than the latter. Consider, e.g., $X=L_{\infty}\left(\mathbb{R}^{d}\right)$. Then $L_{\infty}^{0}$ consists of all functions uniformly continuous on $\mathbb{R}^{d}$, while $L_{\infty}^{+}=\{0\}$.

Now let $S \subset \mathbb{R}^{d}$ be of positive measure. By $X(S)$ we denote the lattice of measurable functions $f$ on $S$ whose extension by zero (denoted by $f_{S}$ ) belongs to $X$. The $F$-norm is given by

$$
\|f ; S\|:=\left\|f_{S}\right\|, \quad f \in X(S) .
$$

Definition 1.9 is then modified as follows.

Definition 1.12. A function $f \in X(S)$ is continuous in this space if

$$
\left\|\left(\tau_{y} f-f\right) \cdot \mathbb{1}_{S \cap\{S+y\}}\right\|=0 .
$$

Clearly, $S \cap\{S+y\}$ is the domain of $\tau_{y} f-f$.

\section{§2. MAin REsults, Formulations}

We begin with notions used in the first compactness criterion. Throughout the remaining part of the paper, $(X,\|\cdot\|)$ stands for a translation-invariant $F$-lattice on $\mathbb{R}^{d}$ and $\Sigma$ for its subset.

Definition 2.1. $\Sigma$ is said to be equicontinuous in $X$ if

$$
\lim _{y \rightarrow 0} \sup _{f \in \Sigma}\left\|\tau_{y} f-f\right\|=0 .
$$


For $X(S)$ this definition is modified in accordance with Definition 1.12, Now, a set $\Sigma \subset X(S)$ is said to be equicontinuous if

$$
\lim _{y \rightarrow 0} \sup _{f \in \Sigma}\left\|\left(\tau_{y} f-f\right) \cdot \mathbb{1}_{S \cap\{S+y\}}\right\|=0 .
$$

Clearly, $\Sigma \subset X^{0}$ in this case.

Definition 2.2. $\Sigma$ is said to be norm absolutely equicontinuous if for every sequence $\left\{S_{n}\right\}$ of measurable sets with empty intersection we have

$$
\lim _{n \rightarrow \infty} \sup _{f \in \Sigma}\left\|f \cdot \mathbb{1}_{S_{n}}\right\|=0 \text {. }
$$

Clearly, $\Sigma$ belongs to $X^{+}$, see Remark 1.11

Theorem 2.3. Let $X \in \mathcal{L}$, see Definition 1.8. Then $\Sigma$ is precompact if and only if $\Sigma$ is bounded, equicontinuous, and norm absolutely equicontinuous.

We recall that the boundedness of $\Sigma$ in the $F$-space $X$ means that for every $\varepsilon>0$ there is $b_{\varepsilon}>0$ such that $\sup _{g \in \Sigma}\left\|b_{\varepsilon} g\right\|<\varepsilon$.

Unfortunately, the condition that is stated above is difficult to verify. We guess it may be replaced by a much easier condition that is stated below.

Definition 2.4. $\Sigma$ is said to vanish at infinity if

$$
\lim _{n \rightarrow \infty} \sup _{f \in \Sigma}\left\|f \cdot \mathbb{1}_{\{|x| \geq n\}}\right\|=0
$$

Hereafter, $|x|:=\max _{1 \leq i \leq d}\left|x_{i}\right|, x \in \mathbb{R}^{d}$.

Conjecture 2.5. $\Sigma$ is precompact in $X \in \mathcal{L}$ if $\Sigma$ is bounded, equicontinuous, and vanishes at infinity.

Here, the necessity of the first condition is clear while the necessity of the others follows from Theorem 2.3 .

We shall prove this conjecture for Orlicz classes, i.e., the following is true.

Theorem 2.6. A subset $\Sigma \subset N(L)$ is precompact if and only if $\Sigma$ is bounded, equicontinuous, and vanishes at infinity in this space.

For Banach lattices, Conjecture 2.5 is true even in a stronger form.

Theorem 2.7. Let $X$ be a Banach lattice of locally integrable functions on $\mathbb{R}^{d}$ belonging to $\mathcal{L}$ and containing the indicators of all subsets of finite measure. Then $\Sigma \subset X$ is precompact if and only if $\Sigma$ is equicontinuous and vanishes at infinity.

If, e.g., supp $X$ is bounded, only the first condition is needed.

It is natural to ask whether the boundedness condition may be discarded in the $F$ lattice case.

The next example demonstrates that, in general, the answer is negative.

Example 2.8. Let $\Sigma:=\left\{n \cdot \mathbb{1}_{(0,1)^{d}}\right\}_{n \in \mathbb{N}}$ be regarded as a subset of $L_{0}$. Clearly, $\Sigma$ is equicontinuous and vanishes at infinity in this space. However, $\Sigma$ is not bounded, hence, is not precompact in $L_{0}$. In fact, if for every $\varepsilon>0$ there exists $b_{\varepsilon}>0$ such that $\sup _{f \in \Sigma}\left\|b_{\varepsilon} f\right\|_{0}<\varepsilon$, then $\left\|b_{\varepsilon} \cdot \mathbb{1}_{(0,1)^{d}}\right\|<\varepsilon$.

But for all $b>0$ the left-hand side equals $N_{0}(1)=1 / 2$.

Nevertheless, for some quasi-Banach lattices the result discussed does hold. 
To present the corresponding result, we introduce the generalized Orlicz class $N(X)$ by replacing $L\left(\mathbb{R}^{d}\right)$ in Example 1.6 (a) with a Banach lattice $X$ of class $\mathcal{L}$. Hence, in this case, the $F$-quasinorm is given by

$$
\rho_{N(X)}(f):=\|N(|f|)\|_{X}
$$

as in the case of $N(L)$, see [21, III. 3], condition (1.9) is sufficient (and necessary) for $N(X)$ to be locally bounded. Therefore, up to renormalization, $N(X)$ is quasi-Banach in this case.

Theorem 2.9. Let $N(X)$ be quasi-Banach and $N$ concave. Then a subset $\Sigma \subset N(X)$ is precompact in this space if and only if $\Sigma$ is equicontinuous and vanishes at infinity.

Corollary 2.10. Consider a Marcinkiewicz-Lorentz space $L_{p, N}\left(\mathbb{R}^{d}\right)$ with $0<p \leq 1$ such that the function $t \mapsto \int_{0}^{t} \frac{N(s)^{p}}{s} d s, t>0$, is finite, concave, and equal to $+\infty$ at infinity. Then $\Sigma \subset L_{p, N}\left(\mathbb{R}^{d}\right)$ is precompact in this space if and only if $\Sigma$ is equicontinuous and vanishes at infinity.

For example, $L_{p, q}\left(\mathbb{R}^{d}\right)$ satisfies these assumptions if $0<p \leq 1$ and $p \leq q<\infty$. We guess that this is also true for $0<q<p$.

Conjecture 2.11. Let $X$ be a quasi-Banach lattice of class $\mathcal{L}$. Then $\Sigma$ is precompact in $X$ if and only if $\Sigma$ is equicontinuous and vanishes at infinity.

The last two theorems are consequences of a Kolmogorov type compactness criterion for Banach lattices.

To formulate it, we define an averaging operator $T_{r}: L^{\text {loc }} \rightarrow L_{\infty}^{\text {loc }}$ by

$$
T_{r} f:=\frac{1}{\left|Q_{r}\right|} \int_{Q_{r}} \tau_{y} f d y,
$$

where $Q_{r}$ stands throughout for the $d$-cube $\left\{x \in \mathbb{R}^{d}:|x|<r\right\}$, while $|x|:=\max _{1 \leq i \leq d}\left|x_{i}\right|$.

Theorem 2.12. Let $\Sigma$ be a subset of a Banach lattice $X$ satisfying the conditions of Theorem 2.7. Then $\Sigma$ is precompact in $X$ if and only if $\Sigma$ vanishes at infinity and

$$
\lim _{r \rightarrow 0} T_{r} f=f
$$

uniformly in $f \in \Sigma$.

Remark 2.13. For a Kolomogorov type criterion, the key point of the proof is the existence of compact linear operators acting in Banach spaces. In general, for quasi-Banach spaces and $F$-spaces, such operators may fail to exist. For example, there are no such operators acting from $L_{p}, p<1$, to any linear topological space; see [10].

\section{§3. ProOFs}

In accordance with logical interrelations between the results, we arrange their proofs in an order deviating from that in $\S 2$.

Proof of Theorem 2.6. We begin with the case of the trace $N(L)(Q)$ of $N(L)$ to a cube $Q$. The corresponding $F$-quasinorm $\|f ; Q\|_{N}$ is defined by

$$
\|f ; Q\|_{N}:=\int_{Q} N(|f|) d x
$$

Proposition 3.1. A subset $\Sigma \subset N(L)(Q)$ is precompact if $\Sigma$ is bounded and equicontinuous. 
Proof. Since $N(L)(Q)$ is a metric space, we can use the Hausdorff compactness criterion to prove precompactness. Hence, given $\varepsilon>0$, a finite $\varepsilon$-net in $\Sigma$ should be found. The next lemmas construct such a net step by step. In their proofs, $Q$ and $K$ denote cubes homothetic to $[0,1]^{d}$.

For $f \in L_{0}$, we set

$$
E(f ; Q):=\inf _{c \in \mathbb{R}} \int_{Q} N(|f-c|) d x
$$

and denote by $c_{Q}(f)$ an optimal $c$ in (3.2).

Lemma 3.2. We have

$$
E(f ; Q) \leq \int_{|y|<2} d y \int_{Q_{r y}} N(|f(x+y)-f(x)|) d x
$$

here $2 r$ is the side length of $Q$ and

$$
Q_{z}:=Q \cap\{Q+z\}=\{x \in Q ; x+z \in Q\} .
$$

Proof. In the iterated integral

$$
I:=\int_{Q} d y \int_{Q} N(|f(x)-f(y)|) d y
$$

we set $u:=x-y, v:=y$ and change the order of integration; we obtain the identity

$$
I=\int_{Q-Q} d u \int_{Q_{u}} N(|f(u+v)-f(v)|) d v .
$$

Setting here $u:=r y, v:=x$ and observing that $Q-Q=(-2 r, 2 r)^{d}$, we conclude that

$$
I=(2 r)^{d} \int_{(-2,2)^{d}} d y \int_{Q_{r y}} N(|f(x+r y)-f(x)|) d x .
$$

On the other hand, by (3.1) and the definition of $I$,

$$
I \geq|Q| \inf _{y \in Q} \int_{Q} N(|f(x)-f(y)|) d y \geq(2 r)^{d} E(f ; Q) .
$$

Combining these formulas, we get the result.

Now, let $\pi_{n}$ be the partition of the cube $Q$ into $n^{d}$ congruent subcubes. We set

$$
s_{n}(f ; Q):=\sum_{K \in \pi_{n}} c_{\stackrel{\circ}{K}}(f) \cdot \mathbb{1}_{K}^{\circ} .
$$

Lemma 3.3. We have

$$
\int_{Q} N\left(\left|f-s_{n}(f ; Q)\right|\right) d x \leq \int_{|y|<2} d y \int_{Q_{\lambda y}} N(|f(x+\lambda y)-f(x)|) d x
$$

where $\lambda:=\frac{r}{n}$.

Proof. Summing in (3.3) over the open cubes $\stackrel{\circ}{K}$ and observing that all $\stackrel{\circ}{K}_{\lambda y}, K \in \pi_{n}$, are included in $Q_{\lambda y}$ and do not intersect, we get (3.6).

Lemma 3.4. The set

$$
S_{n}(\Sigma ; Q):=\left\{s_{n}(f ; Q) ; f \in \Sigma\right\}
$$

is bounded in $N(L)(Q)$. 
Proof. By definition,

$$
s_{n}(c f ; Q)=c s_{n}(f ; Q)
$$

for all constants $c$. This and the $\Delta_{2}$-condition for $N$ together with (3.6) imply the inequality

$$
\int_{Q} N\left(\left|c s_{n}(f ; Q)\right|\right) d x \leq \Delta_{N}\left[\int_{Q} N(c|f|) d x+\int_{|y|<2} d y \int_{Q_{\lambda y}} N(c|f(x+\lambda y)-f(x)|) d x\right] .
$$

The second summand is at most

$$
4^{d} \Delta_{N}\left(\sup _{|y|<2} \int_{Q_{\lambda y}} N(c|f(x+\lambda y)|) d x+\int_{Q} N(c|f|) d x\right) .
$$

By definition, see (3.4), we have $Q_{\lambda y}+\lambda y \subset Q$; hence, this summand is bounded by $2^{2 d+1} \int_{Q} N(c|f|) d x$, whence

$$
\int_{Q} N\left(\left|c s_{n}(f ; Q)\right|\right) d x \leq\left(2^{2 d+1}+1\right) \Delta_{N}^{2} \int_{Q} N(c|f|) d x .
$$

Since $\Sigma$ is bounded in $N(L)(Q)$, for any $\varepsilon>0$ there is $c_{\varepsilon}>0$ such that the right-hand side in (3.8) becomes less than $\varepsilon$ if $c=c_{\varepsilon}$ and $f \in \Sigma$. The same is true for $s_{n}(f ; Q)$, $f \in \Sigma$, i.e., $S_{n}(\Sigma ; Q)$ is bounded in $N(L)(Q)$.

Now we show that $\left\{S_{n}(\Sigma ; Q)\right\}_{n \in \mathbb{N}}$ approximates $\Sigma$ as $n \rightarrow \infty$.

Lemma 3.5. We have

$$
\lim _{n \rightarrow \infty} \sup _{f \in \Sigma} \inf _{s \in S_{n}(\Sigma ; Q)}\|f-s ; Q\|_{N}=0
$$

Proof. By (3.6), the quantity under the limit sign is at most

$$
4^{d} \sup _{|z| \leq \frac{2 r}{n}} \sup _{f \in \Sigma}\left\|\left(\tau_{z} f-f\right) \cdot \mathbb{1}_{Q \cap\{Q+z\}}\right\|_{N} .
$$

By the equicontinuity of $\Sigma$ in $N(L)(Q)$, this tends to 0 as $n \rightarrow \infty$.

The next result will be used many times; therefore, we state it in a fairly general form.

Lemma 3.6. Let $(M, d)$ be a metric space. Let $\Sigma, \Sigma_{n}, n \in \mathbb{N}$, be subsets of $M$ such that

$$
d\left(\Sigma, \Sigma_{n}\right)=\sup _{m \in \Sigma} \inf _{m_{n} \in \Sigma_{n}} d\left(m, m_{n}\right) \rightarrow 0 \quad \text { as } n \rightarrow \infty .
$$

If every $\Sigma_{n}$ is precompact, then $\Sigma$ is also precompact.

Proof. Take $n$ so large that $d\left(\Sigma, \Sigma_{n}\right)<\varepsilon$, and let $G_{n}$ be a finite $\varepsilon$-net for $\Sigma_{n}$. Then $G_{n}$ is a $2 \varepsilon$-net for $\Sigma$.

It remains to show that the set $S_{n}(\Sigma ; Q)$ is compact in $N(L)(Q)$.

In fact, it is contained in the $n^{d}$-dimensional subspace, say $H_{n}$, of $N(L)(Q)$ formed by the step functions generated by the partitions $\pi_{n}$. Since $S_{n}(\Sigma ; Q)$ is bounded in $N(L)(Q)$, it is also bounded in $H_{n}$. Every bounded subset of a finite-dimensional linear topological space is precompact there. Hence, $S_{n}(\Sigma ; Q)$ is precompact in $H_{n}$ and therefore in $N(L)(Q)$.

Proposition 3.1 is proved.

Now we are ready to prove Theorem 2.6. 
Proof of Theorem 2.6. The "if" part. Due to vanishing at infinity, given $\varepsilon>0$, for $\Sigma \subset N(L)$ we can find a cube $Q=Q_{\varepsilon}$ such that

$$
\sup _{f \in \Sigma} \rho_{N}\left(f \cdot \mathbb{1}_{Q^{c}}\right)<\varepsilon .
$$

Now, let $\left\{f_{n}\right\}_{n \in \mathbb{N}} \subset \Sigma$. Write $f_{n}=\mathbb{1}_{Q} \cdot f_{n}+\mathbb{1}_{Q^{c}} \cdot f_{n}=: f_{n}^{1}+f_{n}^{2}$. Since $\left\{f_{n}^{1}\right\} \subset N(L)(Q)$, Proposition 3.1 implies the convergence of some its subsequence in this space. Changing enumeration, we assume that $\left\{f_{n}^{1}\right\}$ converges.

By the triangle inequality for $\rho_{N}$, see Example 1.6 (a), we get

$$
\rho_{N}\left(f_{n}-f_{m}\right) \leq \Delta_{N}\left(\left\|f_{n}^{1}-f_{m}^{1} ; Q\right\|_{N}+\sup _{f \in \Sigma} \rho_{N}\left(f \cdot \mathbb{1}_{Q^{c}}\right)\right) .
$$

Given $\varepsilon>0$, we choose $n_{\varepsilon}$ so that for all $n, m \geq n_{\varepsilon}$ the first summand here becomes at most $\varepsilon$ and estimate the second one by (3.10). This implies

$$
\lim _{n, m \rightarrow \infty} \rho_{N}\left(f_{n}-f_{m}\right)=0
$$

as required.

The "only if" part. The boundedness of $\Sigma$ follows from its precompactness.

Next, since $N(L)$ belongs to the class $\mathcal{L}$, every its element is continuous, see Proposition 1.10, and norm absolutely equicontinuous. Therefore, for a finite $\varepsilon$-net $\Sigma_{\varepsilon}$ of $\Sigma$, given $\varepsilon>0$, we can find $\delta=\delta_{\varepsilon}>0$ and a cube $Q=Q_{\varepsilon}$ such that

$$
\sup _{f \in \Sigma_{\varepsilon}} \rho_{N}\left(\tau_{y} f-f\right)<\varepsilon \quad \text { if }|y|<\delta,
$$

and moreover,

$$
\sup _{f \in \Sigma_{\varepsilon}} \rho_{N}\left(f \cdot \mathbb{1}_{Q^{c}}\right)<\varepsilon .
$$

Since $\Sigma_{\varepsilon}$ is $\varepsilon$-close to $\Sigma$ in $N(L)$, these inequalities are true for $\Sigma$ with $\varepsilon$ replaced by $c_{N} \varepsilon$, where $c_{N}>1$.

Hence, $\Sigma$ is bounded, equicontinuous, and vanishes at infinity.

This proves Theorem 2.6 .

Proof of Theorem 2.3. The "if" part. Let $\Sigma$ be bounded, equicontinuous, and norm absolutely equicontinuous in $X$. We show that every sequence $\left\{f_{n}\right\} \subset \Sigma$ contains a subsequence convergent in $X$.

For this, we present $\Sigma$ as

$$
\Sigma=\Sigma_{1}+\Sigma_{2}:=\left\{f \cdot \mathbb{1}_{Q} ; f \in \Sigma\right\}+\left\{f \cdot \mathbb{1}_{Q^{c}} ; f \in \Sigma\right\} .
$$

Since $\Sigma$ is norm absolutely equicontinuous, there is $Q:=Q_{n_{\varepsilon}}$ such that

$$
\sup _{f \in \Sigma}\left\|f \cdot \mathbb{1}_{Q^{c}}\right\|<\varepsilon .
$$

Regarding $\Sigma_{1}$ as a subset of $X(Q)$ and observing that the linear operator $R_{Q}: f \mapsto$ $f \cdot \mathbb{1}_{Q}$ maps $X$ into $X(Q)$ continuously, we conclude that $R_{Q}(\Sigma)=\Sigma_{1}$ is bounded and equicontinuous in $X(Q)$ if $\Sigma$ is (in $X$ ).

Moreover, by Proposition $1.2(\mathrm{a}), X(Q)$ is continuously embedded into $L_{0}(Q)$. Hence, $\Sigma_{1}$ is bounded and equicontinuous there. Due to Proposition 3.1 with $N(t)=N_{0}(t):=$ $\frac{t}{1+t}, t>0$, the set $\Sigma_{1}$ is precompact in $L_{0}(Q)$.

Hence, the sequence $\left\{f_{k} \cdot \mathbb{1}_{Q}\right\}_{k \in \mathbb{N}} \subset \Sigma$ contains a subsequence convergent in $L_{0}(Q)$. Changing enumeration, we assume that $\left\{f_{n} \cdot \mathbb{1}_{Q}\right\}_{n \in \mathbb{N}}$ itself converges in $L_{0}(Q)$.

Now, by Proposition 1.2 (b), there exists an increasing sequence $\left\{S_{n}\right\}_{n \in \mathbb{N}}$ of measurable sets in $\mathbb{R}^{d}$ such that $\cup S_{n}=\operatorname{supp} X$ and $\mathbb{1}_{S_{n}} \in X$ for all $n$. 
Setting $T_{n}:=\left(Q \backslash S_{n}\right) \cap \operatorname{supp} X$ and using the norm absolute equicontinuity of $\Sigma$, we get

$$
\sup _{f \in \Sigma}\left\|f \cdot \mathbb{1}_{T_{n}}\right\|<\varepsilon
$$

for some $n=\widetilde{n}_{\varepsilon}$.

Since $\left\{f_{k} \cdot \mathbb{1}_{Q}\right\}_{k \in \mathbb{N}}$ converges in $L_{0}(Q)$, given $\varepsilon>0$ there is $k_{\varepsilon}$ such that for all $k, l \geq k_{\varepsilon}$ and $n=\widetilde{n}_{\varepsilon}$ we have

$$
\left|\left\{x \in Q \cap\left(S_{n} \backslash T_{n}\right) ;\left|f_{k}-f_{l}\right|(x)>\varepsilon\right\}\right|<\frac{\varepsilon}{\left\|\mathbb{1}_{S_{n}}\right\|} .
$$

For $k, l \geq k_{\varepsilon}$, the above inequality and formulas (3.10) and (3.12) imply the estimate

$$
\begin{aligned}
\left\|f_{k}-f_{l}\right\| & \leq\left\|f_{k}-f_{l} ; Q\right\|+2 \sup _{f \in \Sigma}\left\|f \cdot \mathbb{1}_{Q^{c}}\right\| \\
& \leq\left(\sup _{Q \cap\left(S_{n} \backslash T_{n}\right)}\left|f_{k}-f_{l}\right|\right)\left\|\mathbb{1}_{S_{n}}\right\|+2 \sup _{f \in \Sigma}\left\|f \cdot \mathbb{1}_{T_{n}}\right\|+2 \sup _{f \in \Sigma}\left\|f \cdot \mathbb{1}_{Q^{c}}\right\| \leq 5 \varepsilon .
\end{aligned}
$$

Hence, $\left\{f_{k}\right\}_{k \in \mathbb{N}}$ converges in $X$ and $\Sigma$ is precompact.

The "only if" part. Since $X \in \mathcal{L}$, its elements are continuous and norm absolutely continuous, and the proof repeats line-to-line that of the "only if" part in Theorem 2.6.

Proof of Theorem 2.12. The "if" part. By the assumptions of the theorem, for every cube $Q \subset \mathbb{R}^{d}$ we have

$$
L_{\infty}(Q) \subset X(Q) \subset L(Q)
$$

Since all these spaces are continuously embedded into $L_{0}\left(\mathbb{R}^{d}\right)$, the closed graph theorem implies the continuity of the embeddings.

Next, $\Sigma$ is approximated uniformly by the family of the averaging operators $T_{r}: X \rightarrow X$ as $r \rightarrow 0$, and vanishes at infinity in $X$. From this, we deduce the precompactness of $\Sigma$ in $X$.

For this, we use the next two results due, respectively, to Mazur, see 11, comments to Subsection VI.1], and Sudakov [24].

Proposition 3.7. (a) Let $\left\{T_{n}\right\}_{n \in \mathbb{N}}$ be a family of linear operators acting compactly in a Banach space $B$. A subset $V \subset B$ is precompact if it is bounded and

$$
\lim _{n \rightarrow \infty} T_{n} x=x
$$

uniformly in $x \in V$.

(b) Let $\left\{T_{n}\right\}_{n \in \mathbb{N}}$ be a family of linear operators acting continuously in a Banach space $B$ and such that

$$
\sup _{n}\left\|T_{n}\right\|<\infty
$$

Assume that for some $k$ and all $n$ the power $T_{n}^{k}$ is compact and $\lambda=1$ is not an eigenvalue for $T_{n}$. Then a subset $V$ is precompact in $B$ if (3.14) is fulfilled uniformly in $x \in V$.

Proof. (a) The boundedness of $V$ implies the precompactness of $T_{n}(V)$. Hence, $V$ is uniformly approximated by the family $\left\{T_{n}(V)\right\}$ of precompact sets and, therefore, is precompact. See Lemma 3.6.

(b) First, we show that $V$ is bounded. To this end, we recall the well-known result of the F. Riesz-Schauder theory asserting that a linear operator $1-T$ is invertible in $B$ if $T$ is compact and the equation $x-T x=0$ has only a trivial solution. This result was generalized by S. Nikolski [19] to linear operators $T$ with compact power.

We conclude that $\left(1-T_{n}\right)^{-1}$ exists for every $n$ and then derive that $V$ is bounded. 
Actually, by the uniform convergence in (3.14),

$$
\sup _{x \in V}\left\|x-T_{n} x\right\|<1
$$

for sufficiently large $n$. Therefore, for these $n$ we have

$$
\sup _{x \in V}\|x\| \leq\left\|\left(1-T_{n}\right)^{-1}\right\| \sup _{x \in V}\left\|x-T_{n} x\right\|<\infty,
$$

as required.

Now we apply assertion (a) to the family of compact operators $\left\{T_{n}^{k}\right\}_{n \in \mathbb{N}}$, assuming that

$$
\lim _{n \rightarrow \infty} T_{n}^{k} x=x
$$

uniformly in $x \in V$. This assumption follows from the inequality

$$
\left\|x_{n}-T_{n}^{k} x\right\| \leq\left(\sum_{j=0}^{k-1}\left\|T_{n}\right\|^{j}\right)\left\|x-T_{n} x\right\|
$$

combined with (3.14) and (3.15).

Finally, the boundedness of $V$ and (3.16) imply the precompactness of $V$.

Now we apply the above proposition to prove the precompactness of $\Sigma$. For this, we use a family of operators $\left\{R_{n}\right\}_{n \in \mathbb{N}}$ defined on $L^{\text {loc }}\left(\mathbb{R}^{n}\right)$ by the formula

$$
R_{n} f:=T_{r}^{2}\left(\mathbb{1}_{Q_{n}} f\right) \quad \text { with } \quad r:=\frac{1}{n} .
$$

We recall that

$$
T_{r} f:=\frac{1}{\left|Q_{r}\right|} \int_{Q_{r}} f(\cdot+y) d y
$$

and $Q_{s}:=\left\{x \in \mathbb{R}^{d} ;|x| \leq s\right\}$.

Lemma 3.8. (a) $R_{n}$ acts in $X$ and

$$
\left\|R_{n}\right\|=1
$$

(b) We have

$$
\lim _{n \rightarrow \infty} R_{n} f=f
$$

uniformly in $f \in \Sigma$.

(c) $R_{n}$ is compact.

(d) $\lambda=1$ is not an eigenvalue of $R_{n}$.

Proof. (a) The $X$-valued function

$$
\widetilde{f}: f \mapsto f(\cdot+y), \quad y \in \mathbb{R}^{d}
$$

is approximated by finite linear combinations of $X$-valued indicators $\widehat{\mathbb{1}}_{S}$ with $|S|<\infty$ because $X$ belongs to $\mathcal{L}$, see, e.g., [11, IV.3.2]. Hence, $\widetilde{f}$ is strongly measurable; moreover, $\|\widetilde{f}(\cdot)\|(=\|f\|)$ is Lebesgue integrable on any cube. Therefore, $\widetilde{f}$ is locally Bochner integrable. By the well-known property of the Bochner integral and the translation invariance of $X$, we obtain

$$
\left\|\frac{1}{\left|Q_{r}\right|} \int_{Q_{r}} \tilde{f}(y) d y\right\| \leq \frac{1}{\left|Q_{r}\right|} \int_{Q_{r}}\|\widetilde{f}(y)\| d y,
$$

i.e., $T_{r}$ acts in $X$ with norm 1. Clearly, this implies (3.18).

(b) Set $\widetilde{R}_{n} f:=T_{r}\left(\mathbb{1}_{Q_{n}} f\right)$ with $r:=1 / n$. Then

$$
\left\|R_{n} f-f\right\| \leq\left\|T_{r}\left(f-\widetilde{R}_{n} f\right)\right\|+\left\|f-\widetilde{R}_{n} f\right\| \leq 2\left\|f-\widetilde{R}_{n} f\right\| .
$$


By (3.17) and (3.18), the last quantity is dominated by $\left\|T_{n^{-1}} f-f\right\|+\left\|f \cdot \mathbb{1}_{Q_{n}^{c}}\right\|$.

By assumption, $\Sigma$ is uniformly approximated by the family $\left\{T_{r}\right\}$ as $r \rightarrow 0$ and vanishes at infinity. Hence, the two terms on the right-hand side tend to 0 as $n \rightarrow \infty$ uniformly in $f \in \Sigma$, i.e., (3.19) holds true.

(c) Let $B_{X}$ be the unit ball of $X$. We must prove the precompactness of $R_{n}\left(B_{X}\right)$. First, we show that the operator acts compactly from $X$ into $C\left(Q_{n+r}\right)$, i.e., $R_{n}\left(B_{X}\right)$ is precompact in the latter space.

Since $R_{n}=T_{r}^{2} \circ \mathbb{1}_{Q_{n}}$ with $r=\frac{1}{n}$, we start with computing the first factor here. By definition, $T_{r} f$ is the convolution of $f$ and $\frac{1}{\left|Q_{r}\right|} \mathbb{1}_{Q_{r}}$; therefore, $T_{r}^{2} f$ is the convolution of $f$ with the kernel

Direct computation gives

$$
v_{r}:=\frac{1}{\left|Q_{r}\right|^{2}} \mathbb{1}_{Q_{r}} * \mathbb{1}_{Q_{r}}
$$

$$
v_{r}(x):=\frac{1}{\left|Q_{r}\right|^{2}} \prod_{i=1}^{d}\left(2 r-\left|x_{i}\right|\right)_{+}, \quad x \in \mathbb{R}^{d},
$$

where $s_{+}:=\max \{s, 0\}$.

Hence, $\operatorname{supp} v_{r}=[-2 r, 2 r]^{d}=Q_{r}$, and for $x, x^{\prime} \in \mathbb{R}^{d}$ we have

$$
\left|v_{r}(x)-v_{r}\left(x^{\prime}\right)\right| \leq d(2 r)^{d-1} \max _{i}\left|x_{i}-x_{i}^{\prime}\right|:=c(r, d)\left|x-x^{\prime}\right| .
$$

This implies

$$
\begin{aligned}
\left|T_{r}^{2} f(x)-T_{r}^{2} f\left(x^{\prime}\right)\right| & =\frac{1}{\left|Q_{r}\right|^{2}}\left|\int_{Q_{r}(x) \cup Q_{r}\left(x^{\prime}\right)}\left[v_{r}(x-y)-v_{r}\left(x^{\prime}-y\right)\right] f(y) d y\right| \\
& \leq \frac{c(d, r)}{r^{2 d}}\left|x-x^{\prime}\right| \int_{Q_{r}(x) \cup Q_{r}\left(x^{\prime}\right)}|f| d y .
\end{aligned}
$$

Since $Q_{r}(x) \cup Q_{r}\left(x^{\prime}\right) \subset Q_{n+r}$ whenever $x, x^{\prime} \in Q_{n}$, for these $x, x^{\prime}$ we get

$$
\left|T_{r}^{2} f(x)-T_{r}^{2} f\left(x^{\prime}\right)\right| \leq 2 d r\left|x-x^{\prime}\right| \int_{Q_{n+r}}|f| d y .
$$

Then for $R_{n} f:=T_{r}^{2}\left(f \cdot \mathbb{1}_{Q_{n}}\right)$ we deduce that

$$
\left|R_{n} f(x)-R_{n} f\left(x^{\prime}\right)\right| \leq 2 d r\left|x-x^{\prime}\right| \int_{Q_{n+r}}|f| d y
$$

for $x, x^{\prime} \in Q_{n}$. Since $X$ is continuously embedded into $L\left(Q_{n+r}\right)$, see (3.13), the integral on the right-hand side is at most $c(n+r)\|f\| \leq c(n+r)$ because $f \in B_{X}$; hence,

$$
\left|R_{n} f(x)-R_{n} f\left(x^{\prime}\right)\right| \leq 2 c(n+r) d r\left|x-x^{\prime}\right|
$$

for $x, x^{\prime} \in Q_{n}$.

Moreover, $R_{n}\left(B_{X}\right)$ is bounded in $C\left(Q_{n+r}\right)$, because for $x \in Q_{n}$ and $f \in B_{X}$ we have

$$
\left|R_{n} f(x)\right| \leq\left(\sup v_{1 / n}\right) \int_{Q_{n+1 / n}}|f| d x \leq c(n)\|f\| \leq c(n) .
$$

This and the preceding inequality imply that $R_{n}\left(B_{X}\right)$ satisfies the conditions of the Arcela-Ascoli theorem, i.e., is precompact in $C\left(Q_{n+r}\right)$.

Using again (3.13), we conclude that $C\left(Q_{n+r}\right)$ is continuously embedded in $X$. Hence, $R_{n}\left(B_{X}\right)$ is also precompact in $X$, and $R_{n}$ acts compactly in $X$.

(d) We must show that the equation

$$
R_{n} f=f
$$

has only a trivial solution. 
The following simple argument is due to Sudakov [24]1. Since the function $R_{n} f:=$ $T_{r}^{2}\left(f \cdot \mathbb{1}_{Q_{n}}\right)$ is continuous and vanishes outside $Q_{n+r}$, a nontrivial solution achieves extreme values inside $Q_{n+r}$ and at least one of them, say $x_{0}$, differs from 0 . Let for definiteness $\max f>0$ and $S:=\left\{x \in Q_{n+r} ; f(x)=\max f\right\}$. If $x_{0}$ is a boundary point of this closed set, then $Q_{r}\left(x_{0}\right) \backslash S$ contains a closed subset $F \subset Q_{2 r}\left(x_{0}\right)$ of positive measure such that for some $q, 0<q<1$, we have

$$
f(x)<q \max f=q f\left(x_{0}\right), \quad x \in F .
$$

Setting for brevity $d \mu:=\frac{1}{\left|Q_{r}\right|^{2}} v_{r}\left(r-x_{0}\right) d x$ and $Q:=Q_{2 r}\left(x_{0}\right)$, we get

$$
\begin{aligned}
f\left(x_{0}\right)=\left(R_{n} f\right)\left(x_{0}\right) & =\int_{Q \backslash F} f \cdot \mathbb{1}_{Q_{n}} d \mu+\int_{F} f \cdot \mathbb{1}_{Q_{n}} d \mu \\
& \leq f\left(x_{0}\right) \mu(Q \backslash F)+q f\left(x_{0}\right) \mu(F)<f\left(x_{0}\right) \mu(Q)=f\left(x_{0}\right),
\end{aligned}
$$

a contradiction.

Lemma 3.8 is proved.

Combining this result and Proposition 3.7 we conclude that the assumptions of Theorem 2.12 are sufficient for the precompactness of $\Sigma$.

The "only if" part. Since $X \in \mathcal{L}$, the proof repeats that of the "only if" part for Theorem 2.6.

Theorem 2.12 is proved.

Proof of Theorem 2.7. The "if" part follows directly from that of Theorem 2.12. Actually, if $\Sigma$ is equicontinuous and vanishes at infinity in $X \in \mathcal{L}$, then as above

$$
\sup _{f \in \Sigma}\left\|f-T_{r} f\right\| \leq \sup _{f \in \Sigma} \sup _{|y| \leq r}\left\|\tau_{y} f-f\right\| .
$$

Since the right-hand side tends to 0 as $r \rightarrow 0$, the assumptions of Theorem 2.12 are fulfilled for $\Sigma$, i.e., $\Sigma$ is precompact.

The "only if" part $\Sigma$ was, in fact, established within the proof of Theorem 2.6

Proof of Theorem 2.9. By the definition of $N(X)$, the map $f \mapsto N(|f|)$ acts from $N(X)$ into $X$. We denote the image of $\Sigma \subset N(X)$ by $N(\Sigma)$ and show that it is equicontinuous and vanishes at infinity, i.e., is precompact in $X$ by Theorem 2.7 .

By the concavity of $N$, we get

$$
\left\|N\left(\left|\tau_{y} f\right|\right)-N(|f|)\right\|_{X} \leq\left\|N\left(\left|\tau_{y} f-f\right|\right)\right\|_{X}=: \rho_{N(X)}\left(\tau_{y} f-f\right) .
$$

Hence, $N(\Sigma)$ is equicontinuous in $X$, because so is $\Sigma$ in $N(X)$.

Next, since $N>0$ on $(0,+\infty)$, we have

$$
\mathbb{1}_{S} \cdot N(|f|)=N\left(\left|\mathbb{1}_{S} \cdot f\right|\right) .
$$

We conclude that $N(\Sigma)$ vanishes at infinity in $X$, because so does $\Sigma$ in $N(X)$.

Now, by the "only if" part of Theorem [2.3, the precompactness of $N(\Sigma)$ in $X \in \mathcal{L}$ implies its norm absolute equicontinuity and boundedness in $X$. This, in its turn, implies the same for $\Sigma$ in $N(X)$.

In fact, for a sequence $\left\{S_{n}\right\}_{n \in \mathbb{N}}$ with $\bigcap S_{n}=\varnothing$, by (3.21) we get

$$
\lim _{n \rightarrow \infty} \sup _{f \in \Sigma}\left\|\mathbb{1}_{S_{n}} \cdot f\right\|_{N(X)}=\lim _{n \rightarrow \infty} \sup _{f \in \Sigma}\left\|\mathbb{1}_{S_{n}} \cdot N(|f|)\right\|_{X}=0
$$

\footnotetext{
${ }^{1}$ It is used here for the operator $\widetilde{R}_{n}$, see (b), whose square was claimed in 24 to be compact in $L\left(\mathbb{R}^{d}\right)$, which would have ensured the applicability of Proposition 3.7 with $k=2$ to $\left\{\widetilde{R}_{n}\right\}$. The claim is incorrect, but the argument can be adapted to our case.
} 
and moreover,

$$
\sup _{f \in \Sigma}\|f\|_{N(X)}:=\sup _{f \in \Sigma}\|N(|f|)\|_{X}<\infty .
$$

Finally, $N(X) \in \mathcal{L}$ because $X \in \mathcal{L}$, while $\Sigma$ is bounded, vanishes at infinity, and is norm absolutely equicontinuous in $N(X)$. Then, using the "if" part of Theorem 2.3, we obtain the precompactness of $\Sigma$ in $N(X)$.

Proof of Corollary 2.10. To apply Theorem 2.9. we must present $L_{p, N}$ as a generalized Orlicz space. To this end, we use the identity $\left(|f|^{p}\right)^{*}=\left(f^{*}\right)^{p}$ to write

$$
\|f\|_{p, N}:=\int_{\mathbb{R}_{+}}\left(f^{*} N\right)^{p} \frac{d t}{t}=\int_{\mathbb{R}_{+}}\left(f^{*}\right)^{p} N^{p} \frac{d t}{t}=\left\||f|^{p}\right\|_{L_{1, N} p} .
$$

Hence, $L_{p, N}=N_{p}\left(L_{1, N^{p}}\right)$, where $N_{p}: t \mapsto t^{p}, t>0$, is concave for $0<p \leq 1$.

We show that $L_{1, N^{p}}$ is the desired Banach lattice. For this, we write

$$
\|f\|_{L_{1, N^{p}}}=\int_{\mathbb{R}_{+}} f^{*} N^{p} \frac{d t}{t}=\int_{\mathbb{R}_{+}} f^{*} d \Psi
$$

where

$$
\Psi(t):=\int_{0}^{t} \frac{N(s)^{p}}{s} d s, \quad t>0 .
$$

By the assumptions, $\Psi$ is concave and $\Psi(+\infty)=\infty$.

It is well known, see, e.g., [15, II.5], that the quasinorm on the right-hand side is a Banach norm if $\Psi$ is concave, and the space $L_{1, N^{p}}$ belongs to $\mathcal{L}$ if $\Psi(t) \rightarrow+\infty$ as $t \rightarrow+\infty$.

The proof is complete.

\section{$\S 4$. Concluding Remarks}

4.1. The first compactness result for non-Banach case is due to Fréchet [6] in 1927 for the space $L_{0}(0,1)$; a simpler proof was given in 8 . A special case of Theorem 2.6 with $N(t):=\frac{t}{1+t}, t>0$, gives another compactness criterion for both $L_{0}(Q)$ and $L_{0}\left(\mathbb{R}^{d}\right)$, seemingly more suitable for applications.

4.2. A special case of Theorem 2.6 for $L_{p}$-spaces with $1<p<\infty$ and bounded support was obtained by M. Riesz [20] as a direct consequence of Kolmogorov's compactness criterion [13]. Then Tamarkin [26] and Tulajkov [29] extended the latter, and hence, the former, to $L_{p}$-spaces with unbounded support and to $p=1$, respectively. Finally, Sudakov 24] showed that the boundedness assumption can be discarded for all these results.

It may be of interest to prove Theorem 2.7 directly without the use of the Kolomogorov type criterion of Theorem 2.12

The compactness result for $L_{p}$ with $p<1$ is due to Tsuji [28], whose paper is now available via Internet. In his 1978 lectures in Yaroslavl' university, the present author proved this result by a new approach given in a general form in Proposition 3.1. This was then extended in Nevskii's PhD thesis to quasi-Banach Orlicz spaces [18. In the two cases, compactness was used to prove a Whitney type inequality. A version of this inequality for $L_{p}$ with $p<1$ was recently proved in [9, Appendix] by the use of the very same compactness approach. 
4.3. The class of $B$-lattices in Theorems 2.7 and 2.12 can be widened substantially for those with bounded support; actually, the following is true in this case.

Theorem 2.7'. Let $X$ be a Banach lattice on $\mathbb{R}^{d}$ having bounded support and contained between $L_{\infty}^{\mathrm{loc}}$ and $L^{\mathrm{loc}}$. A subset $\Sigma \subset X^{0}$ is precompact in this space if and only if $\Sigma$ is equicontinuous.

The only essential change in the proof is to define $T_{r}$ by using the Riemann integral. Since for $f \in X^{0}$ the $X$-valued function $y \mapsto f(\cdot+y), y \in \mathbb{R}^{d}$, is continuous, this function is Riemann integrable and, moreover,

$$
\left\|T_{r} f\right\| \leq \frac{1}{\left|Q_{r}\right|} \int_{Q_{r}}\left\|\tau_{y} f\right\| d y
$$

as is required in the proof of Theorem 2.12 (hence, Theorem 2.7) for this case.

Let, e.g., $X=L_{\infty}$. Then $X^{0}$ is the space of uniformly continuous functions on $\mathbb{R}^{d}$ vanishing outside supp $X$. For this case, Theorem $2.7^{\prime}$ gives a version of the Arzela-Ascoli theorem with the boundedness condition replaced by

$$
\lim _{x \rightarrow \infty} \sup _{f \in \Sigma} f(x)=0 .
$$

4.4. A version of Theorem $2.7^{\prime}$ holds true for the spaces $X(S)$, see $\left(2.1^{\prime}\right)$ for the definition, associated with a Banach lattice $X$ on $\mathbb{R}^{d}$, where $S$ is a bounded Lipschitz domain. In this case, the family $\left\{T_{r}\right\}$ is defined by

$$
T_{r} f(x):=\frac{1}{\left|S_{r}(x)\right|} \int_{S_{r}(x)} f(y) d y, \quad x \in S,
$$

where $S_{r}(x):=S \cap Q_{r}(x)$.

However, boundedness is necessary in this version, as the example $\Sigma=\mathbb{R}$ shows. The impossibility to apply now the Riesz-Schauder theory is caused by the existence of nontrivial solutions (constants) for the key equation $T_{r}^{2} f=f$.

4.5. All of the basic notions of the present paper can easily be adapted to the more general setting of a $\sigma$-compact Abelian group with the Haar measure. It can be verified directly that all of the above proofs are valid for this case. In particular, in this way we can obtain the following elegant Shilov criterion [23] for compact Abelian groups. The initial proof requires some powerful means and results of harmonic analysis on compact Abelian groups, presented in detail in [22].

4.6. Suppose $(G,+)$ is a compact Abelian group with the Haar measure $\mu_{H}$. Let $C h(G)$ denote the set of continuous characters for $G$. A Banach space $(X(G),\|\cdot\|)$ of complexvalued $\mu_{H}$-measurable functions on $G$ is said to be homogeneous if the shifts $\tau_{h}: f \mapsto$ $f(\cdot+h), h \in G$, are isometries of $X(G)$ and for every $f \in X(G)$ we have

$$
\lim _{h \rightarrow 0}\left\|\tau_{h} f-f\right\|=0 .
$$

We also assume that

$$
C h(G) \subset X(G) \subset L\left(G, \mu_{H}\right) .
$$

Then the following is true.

Theorem 4.1 (Shilov [23]). A subset $\Sigma \subset X(G)$ is precompact in this space if and only if $\Sigma$ is bounded and equicontinuous. 


\section{$\S 5$. Appendix}

Since the paper 4] is now hardly available, we outline the proof of the Whitney type inequality from there, based on a compactness argument.

Let $(X,\|\cdot\|)$ be a quasi-Banach lattice on a cube $Q \subset \mathbb{R}^{d}$. We say that $X$ belongs to the Arzela-Ascoli class (briefly, $X \in \mathcal{A A}$ ) if every bounded equicontinuous subset of $X$ is precompact. For instance, the spaces $N(X)$ of Theorem 2.9 are of this class.

To formulate the main result, we recall that the $k$-modulus of continuity for $f \in X$ is a function of $t \in\left(0, \frac{\operatorname{diam} Q}{k}\right)$ given by

$$
\omega_{k}(f ; t):=\sup _{|y| \leq t}\left\|\left(\tau_{y}-1\right)^{k} f ; Q_{k y}\right\| .
$$

As above, here $\|\cdot ; S\|$ is the trace quasinorm of $X$ to a subset $S \subset Q, Q_{k y}:=\{x \in$ $Q ;[x, x+k y] \subset Q\}$, and $|y|:=\max _{1 \leq i \leq d}\left|y_{i}\right|$ (in particular, $\operatorname{diam} Q$ equals the side length of $Q$ ).

Next, let $\mathcal{P}_{l}$ be the linear space of polynomials in $x \in \mathbb{R}^{d}$ of degree $l$, and let

$$
E_{k}(f):=\inf _{p \in \mathcal{P}_{k-1}}\|f-p\|
$$

be the best approximation of $f$ by $\left.\mathcal{P}_{k-1}\right|_{Q}$.

We assume that $\mathcal{P}_{l}$ with $l<0$ is $\{0\}$, so that (5.2) is $\|f\|$ if $k=0$.

Theorem 5.1. Let $X$ belong to $\mathcal{A A}$ and contain constants. For any $k \in \mathbb{N}$, there is a constant $c=c\left(k, d, p_{X}\right)>1$ such that for every $f \in X$ we have

$$
E_{k}(f) \leq c \omega_{k}\left(f ; \frac{\operatorname{diam} Q}{k}\right) .
$$

Remark 5.2. We recall that $p_{X}:=\left(\log _{2} c_{X}+1\right)^{-1}$, where $c_{X}$ is the infimum of the constants $c$ in the inequality $\|f+g\| \leq c(|f\|+\mid g\|)$. In particular,

$$
\|f\|^{*} \leq\|f\|^{p} \leq 4\|f\|^{*}
$$

for $0<p<p_{X}$, where $\|\cdot\|^{*}$ is the lattice $p$-norm on $X$ defined in Proposition 1.3 .

Proof. By (5.4) and definitions (5.1) and (5.2), we can and do assume that $\|\cdot\|$ is a $p$-norm. In particular, this implies the inequality

$$
\left|E_{k}(f)-E_{k}(g)\right| \leq E_{k}(f-g)
$$

and the $p$-homogeneity of $E_{k}$ and $\omega_{k}$ with respect to $f$.

Assume to the contrary that (5.3) is not true. Then there exists a sequence $\left\{f_{n}\right\}_{n \in \mathbb{N}}$ in $X$ such that

$$
E_{k}\left(f_{n}\right)=1, \quad \omega_{k}\left(f_{n} ; \frac{\operatorname{diam} Q}{k}\right) \leq \frac{1}{n}, \quad n \in \mathbb{N}
$$

here we use the $p$-homogeneity of these functionals.

Let $p_{k}(f)$ be an optimal polynomial of degree $k-1$ in (5.2). Setting $g_{n}:=f_{n}-p_{k}\left(f_{n}\right)$ and observing that $\left(\tau_{y}-1\right)^{k}$ annihilates $\mathcal{P}_{k-1}$, from (5.6) we deduce that

$$
\left\|g_{n}\right\|=1, \quad \omega_{k}\left(g_{n} ; \frac{\operatorname{diam} Q}{k}\right) \leq \frac{1}{n}, \quad n \in \mathbb{N} .
$$

Since $\omega_{k}$ is monotone nondecreasing in $t$, the inequality implies that

$$
\lim _{t \rightarrow 0} \sup _{n} \omega_{k}\left(g_{n} ; t\right)=0 .
$$

From this we derive that (5.8) is true for $k=1$ as well. 
Lemma 5.3 (Marchaud inequality). There is a constant $c=c(k, p)>1$ such that

$$
\omega_{1}(f ; t) \leq c t\left(\int_{t}^{\frac{\operatorname{diam} Q}{k}} \frac{\omega_{k}(f ; s)}{s^{2}} d s+\|f\|\right), \quad t \in\left(0, \frac{\operatorname{diam} Q}{k}\right) .
$$

For the Banach case (here $p=1$ ) the proof is based on the Marchaud identity, triangle inequality, and the homogeneity of the norm, see, e.g., [17, Subsection 2.8]. In our case, $\|\cdot\|$ is $p$-homogeneous and so the constant in (5.9) depends also on $p$.

\section{Corollary 5.4.}

$$
\lim _{t \rightarrow 0} \sup _{n} \omega_{1}\left(g_{n} ; t\right)=0
$$

Proof. Set

$$
\eta_{k}(t):=\sup _{n} \omega_{k}\left(g_{n} ; t\right)
$$

By (5.9),

$$
\eta_{1}(t) \leq c t\left(\int_{s \geq t} \frac{\eta_{k}(s)}{s^{2}} d s+\sup _{n}\left\|g_{n}\right\|\right)=c t+c t \int_{s \geq t} \frac{\eta_{k}(s)}{s^{2}} d s .
$$

Since $\eta_{k}(t) \rightarrow 0$ as $t \rightarrow 0$, the second term tends to 0 as $t \rightarrow 0$.

This and the identity in (5.7) imply that $\left\{g_{n}\right\}_{n \in \mathbb{N}}$ is bounded and equicontinuous in $X \in \mathcal{A} \mathcal{A}$, hence, is precompact. Changing enumeration, we may assume that $\left\{g_{n}\right\}_{n \in \mathbb{N}}$ converges to some $g$ in $X$. Using (5.5) and a similar inequality for $\omega_{k}$ with $g$ and $g_{n}$ and passing to the limit in (5.7) as $n \rightarrow \infty$, we get

$$
E_{k}(g)=\|g\|=1 \quad \text { and } \quad \omega_{k}\left(g ; \frac{\operatorname{diam} Q}{k}\right)=0 .
$$

We show that the second identity here implies that $g \in \mathcal{P}_{k-1}$, in contradiction to the first identity.

Lemma 5.5. If $\omega_{k}\left(g ; \frac{\operatorname{diam} Q}{k}\right)=0$, then $\left.g \in \mathcal{P}_{k-1}\right|_{Q}$.

Proof. Since $X \subset L_{0}(Q)$, the assumption implies that

$$
\Delta_{h}^{k} g(x)=0 \quad \text { a.e. on } Q_{k h}
$$

for every $h$. By the definition of $\Delta_{h}^{k}$, this yields

$$
|g(y)| \leq \sum_{j=1}^{k}\left(\begin{array}{l}
k \\
j
\end{array}\right)|g(y+j x)|
$$

a.e. for $x$ belonging to the set

$$
Q[k, y]:=\left(k^{-1}(Q-y)\right) \cap Q .
$$

Now, by Kalton's result [10, §8], every $p$-Banach lattice embeds continuously into the Marcinkiewicz-Lorentz space $L_{p \infty}$. Hence, $X$ embeds continuously into any $L_{q}(Q)$ with $0<q<p$; in particular, we can integrate (5.12) in $x$ with power $q$. This implies

$$
|g(y)| \cdot\left\|\mathbb{1}_{Q[k, y]}\right\|_{q} \leq c(q, k) \sum_{j=1}^{k}\left\{\int_{Q[k, y]}|g(y+j x)|^{q} d x\right\}^{\frac{1}{q}} .
$$

Setting $z=y+j x$ in the $j$ th term and observing that $z \in Q$ if $x \in Q[k, y]$, we get

$$
\left\|\mathbb{1}_{Q[k, y]}\right\|_{q}|g(y)| \leq c(q, k)\left(\sum_{j=1}^{k} j^{-d / q}\right)\|g\|_{q} \leq c(q, k, X)\left(\sum_{j=1}^{k} j^{-d / q}\right)\|g\| ;
$$

in the last inequality we have used the continuous embedding of $X$ into $L_{q}(Q)$. 
Next, assume without lost of generality that $Q:=[-1,1]^{d}$. Given a vertex $\varepsilon \in$ $\{-1,1\}^{d}$ of this cube, let $Q^{\varepsilon}$ be a subcube of $Q$ containing 0 and $\varepsilon$ as its vertices. Direct computation shows that $Q[k, y]$ contains a cube of volume $k^{-d}$. Hence, the factor on the left-hand side of (5.14) is at least $k^{-d / q}$. Therefore, (5.14) implies

$$
|g(y)| \leq c(q, k, X)\|g\|, \quad y \in Q^{\varepsilon} \quad \text { a.e. }
$$

Moreover, the union of the cubes $Q^{\varepsilon}$ equals $Q$, whence $g \in L_{\infty}(Q)$.

Finally, using this and (5.11), we write

$$
\left\|\Delta_{h}^{k} g ; Q_{k h}\right\|_{1}=\sup _{\varphi \in L_{\infty}\left(Q_{k h}\right)}\left|\int_{Q_{k h}}\left(\varphi \Delta_{h}^{k} g\right) d x\right|=0 .
$$

Since inequality (5.3) is true for $X=L(Q)$, see [3], we get

$$
E_{k}(g) \leq c(k, d) \sup _{h}\left\|\Delta_{h}^{k} f ; Q_{k h}\right\|_{1}=0
$$

for this space, i.e., $\left.g \in \mathcal{P}_{k-1}\right|_{Q}$ as required.

Since the last lemma and (5.10) lead to a contradiction, Theorem 5.1 is proved.

\section{REFERENCES}

[1] S. Banach, Théorie des opérations linéaires, Monografie Matematyczne, Vol. 1, Warsaw, 1932.

[2] Yu. Brudnyi, Rational approximation and embedding theorems, Dokl. Akad. Nauk SSSR 247 (1979), no. 2, 269-272; English transl., Soviet Math. Dokl. 20 (1979), 681-684. MR545347 (81d:41016)

[3] _ A multidimensional analogue of a certian theorem of Whitney, Mat. Sb. 62 (1970), no. 2, 175-19; English transl., Sb. Math. 11 (1970), 157-170. MR0267319(42:2221)

[4] _ Whitney's type inequality for quasi-Banach spaces, Function Spaces and Applications to Differential Equations, Russian Univ. Peopl. Friendship, Moscow, 1992, pp. 21-27. (Russian)

[5] N. Dunford and J. T. Schwartz, Linear operators. I, General Theory, Pure and Appl. Math., vol. 7, Interscience Publ., Inc., New York, 1958. MR0117523 (22:8302)

[6] M. Fréchet, Sur les ensembles compacts de fonctions mesurables, Fund. Math. 9 (1927), 25-32.

[7] A. H. Frink, Distance functions and the metrization problem, Bull. Amer. Math. Soc. 43 (1937), no. 2, 133-142. MR1563501

[8] E. Hanson, A note on compactness, Bull. Amer. Math. Soc. 39 (1933), no. 6, 397-400. MR1562635

[9] L. Heldberg and Yu. Netrusov, An axiomatic approach to function spaces, spectral synthesis and Luzin approximation, Mem. Amer. Math. Soc. 188 (2007), no. 882. MR2326315 (2008d:46045)

[10] N. Kalton, Compact and strictly singular operators on Orlicz spaces, Israel J. Math. 26 (1977), no. 2, 329-355. MR0636121 (58:30422)

[11] L. V. Kantorovich and G. P. Akilov, Functional analysis, Pergamon Press, Oxford, 1982. MR664597 (83h:46002)

[12] Yu. I. Gribanov, Nonlinear operators in Orlicz spaces, Uchen. Zap. Kazan. Univ. 115 (1955), no. 7, 5-13. (Russian)

[13] A. N. Kolmogoroff, Über die Kompaktheit der Funktionenmengen bei der Konvergenz im Mittel, Nachr. Akad. Wiss. Göttingen Math.-Phys. Kl., 1931, 60-63.

[14] M. A. Krasnoselskii and Ya. B. Rutickii, Convex functions and Orlicz spaces, P. Noordhoff Ltd., Groningen, 1961. MR0126722 (23:A4016)

[15] S. G. Krein, Yu. I. Petunin, and E. M. Semenov, Interpolation of linear operators, Transl. Math. Monogr., vol. 54, Amer. Math. Soc., Providence, RI, 1982. MR649411 (84j:46103)

[16] J. Lindenstrauss and L. Tzafriri, Classical Banach spaces. I, Sequence spaces, Ergebniss Math., vol. 92, Springer-Verlag, Berlin, 1977. MR0500056 (58:17766)

[17] G. G. Lorentz and R. DeVore, Constructive approximation, Grundlehren Math. Wiss., Bd. 303, Springer-Verlag, Berlin, 1993. MR,1261635 (95f:41001)

[18] M. V. Nevskii, Approximation of functions in Orlicz classes, Studies in the Theory of Functions of Several Real Variables, Ed. by Yu. Brudnyi, Yaroslav. Gos. Univ., Yaroslavl', 1984, pp. 83-101. (Russian) MR830221 (87i:41033)

[19] S. M. Nikol'skii, Linear operators in metric spaces, Dokl. Akad. Nauk SSSR 2 (1936), no. 8, $309-312$. (Russian)

[20] M. Riesz, Sur les ensembles compacts de fonctions sommables, Acta. Sci. Math. Szeged 6 (1933), 136-142. 
[21] S. Rolewicz, Metric linear spaces, Monografie Matematyczne, vol. 56, PWN-Polish Sci. Publ., Warszaw, 1972. MR0438074 (55:10993)

[22] G. E. Shilov, Homogeneous rings of functions, Uspekhi Mat. Nauk 6 (1951), no. 1, 91-137. (Russian) $\operatorname{MR} 0042617(13: 139 \mathrm{c})$

[23] Criteria of compactness in homogeneous spaces of function, Dokl. Akad. Nauk SSSR 92 (1953), no. 1, 11-12. (Russian) MR0058864 (15:437e)

[24] V. N. Sudakov, Criteria of compactness in function spaces, Uspekhi Mat. Nauk 12 (1957), no. 3, 221-224. (Russian) MR0090023 (19:756d)

[25] Takahashi T., On the compactness of the function-set by the convergence in mean of general type, Studia Math. 5 (1934), 141-150.

[26] J. D. Tamarkin, On the compactness of the space $L_{p}$, Bull. Amer. Math. Soc. 38 (1932), no. 2, 79-84. MR1562331

[27] A. F. Timan, Theory of approximation of functions of a real variable, Intern. Ser. Monogr. Pure Appl. Math., vol. 34, Pergamon Press, New York, 1963. MR0192238 (33:465)

[28] M. Tsuji, On the compactness of space $L_{p}(p>0)$ and its application to integral operators, Kodai Math. Sem. Rep. 3 (1951), 33-36. MR0043350(13:247e)

[29] A. Tulajkov, Zur Kompaktheit im Raum $L_{p}$ für $p=1$, Nachr. Akad. Wiss. Göttingen, Math.-Phys. Kl., 1933, 167-170.

Department of Mathematics, Technion, 32000, Haifa, IsRael

E-mail address: ybrudnyi@math.technion.ac.il

Received 18/JUL/2012

Originally published in English 